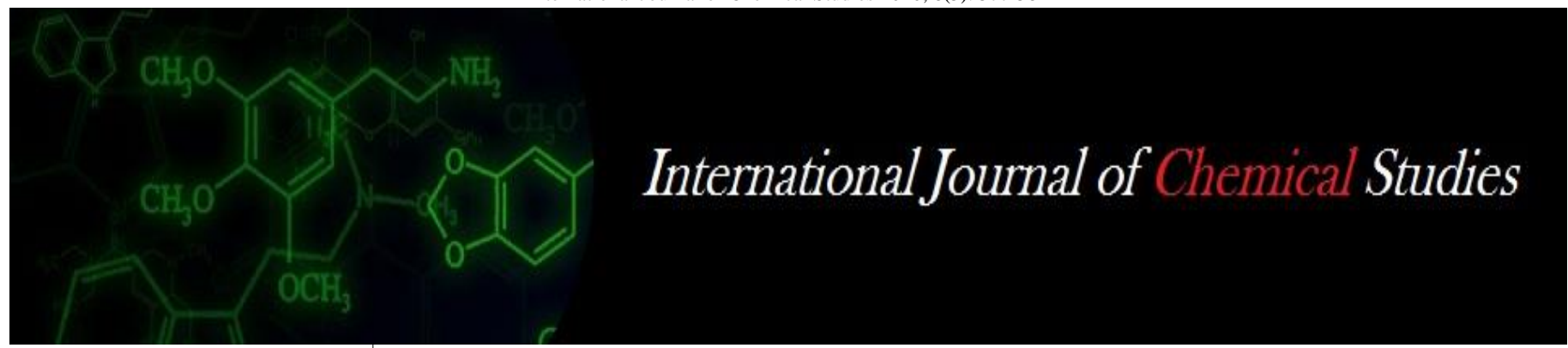

P-ISSN: 2349-8528

E-ISSN: 2321-4902

www.chemijournal.com

IJCS 2020; 8(5): 577-581

(C) 2020 IJCS

Received: 20-07-2020

Accepted: 28-08-2020

\section{RV Hajari}

Ph.D., Student, Department of Agronomy, B.A. College of Agriculture, Anand Agricultural University, Anand, Gujarat, India

\section{Dr. GJ Patel}

Research Scientist, Tribal

Research cum Training Center, Anand Agricultural University, Devgadhbaria, Anand, Gujarat, India
Corresponding Author: RV Hajari

Ph.D., Student, Department of Agronomy, B.A. College of Agriculture, Anand Agricultural University, Anand, Gujarat, India

\section{Pre and post - emergence herbicides with cultural practices on weed management in soybean - pigeonpea intercropping system under middle Gujarat conditions}

\author{
RV Hajari and Dr. GJ Patel
}

DOI: https://doi.org/10.22271/chemi.2020.v8.i5h.10358

\begin{abstract}
To study the "pre and post-emergence herbicides with cultural practices on weed management in soybean-pigeonpea intercropping system under middle Gujarat condition", an experiment was conducted at Agriculture Research Station, Anand Agricultural University, Derol, Dist: panchmahal, Gujarat, India during kharif seasons of the years 2017-18 and 2018-19. Ten treatments for weed management were studied in randomized block design with four replications. Result of field experimentation, it seems quite logical that potential production, profit and economic weed management can be achieved in soybean pigeonpea intercropping system by interculturing + hand weeding at $20 \& 40 \mathrm{DAS}$ or post-emergence application of imazamox 35\% + imazethapyr 35\% (Pre mix) $70 \mathrm{~g} \mathrm{ha}^{-1} \mathrm{PoE} f b$ interculturing + hand weeding at $30 \mathrm{DAS}$.
\end{abstract}

Keywords: Soybean, pigeonpea, weed control, herbicide and soybean equivalent yield

\section{Introduction}

Soybean considered as the 'Miracle crop or Wonder legume'. It has outstanding nutritive value with $43 \%$ biological protein, $20 \%$ oil and is also very rich in vitamins, iron, mineral, salts and amino acids (Sangeetha et al. 2013) ${ }^{[15]}$. Pigeonpea (Cajanus cajan (L.) Millsp.) commonly known as red gram, tur or arhar is the fifth prominent legume crop in the world.It has been recognized as a valuable source of protein for the vegetarians in their daily diet. It is grown as sole crop or intercrop with urdbean, mungbean, castor, sorghum, soybean, cotton, maize and groundnut in different states like Maharashtra, Karnataka, Andhra Pradesh, Gujarat, Jharkhand, Rajasthan Odisha, Punjab and Haryana. In rainy season weeds come in 2-3 flushes and growth is very fast therefore, they compete for light, nutrient, and space and are responsible for considerable reduction in yield. Weeds caused $80 \%$ reduction in pigeonpea grain yield if weeds were allowed to grow till harvest, however, grain yield losses were only $38 \%$ in pigeonpea + soybean intercropping system (Talnikar et al. 2008) ${ }^{[19]}$.

Intercropping is the agricultural practice of growing two or more crops simultaneously on the same field with a definite row proportion. Weed suppression, the reduction of weed growth by crop interference, has been referred as one determinant of yield advantage of intercropping. The first 20-40 days period after sowing of soybean is considered to be critical with respect to weed crop competition. Weed competition during this period may lead to 40-70\% reduction in the seed yield of soybean (Bhan, 1975) ${ }^{[2]}$ depending upon the weed species present. Weeds are known to cause $40-64 \%$ reduction in pigeonpea yield. The critical period of crop weed competition for pigeonpea is 4-8 weeks (Ali, 1992) ${ }^{[1]}$. Among various constraints in crop production weed control is important one but generally neglected by many farmers. The crop yield loss is 20 to 77 per cent due to weeds (Kurchania et al. 2001) ${ }^{[10]}$. Inter cropping suppress the growth of weeds up to $25 \%$ (Sobney et al. 1989) ${ }^{[17]}$. It is very essential to find out alternative to manual labour for weed control, which has more weed control efficiency. At present many promising and selective herbicides are available which can control weeds effectively. Therefore, the present study was conducted to study the pre and post-emergence herbicides with cultural practices on weed management in soybean - pigeonpea intercropping system under middle Gujarat condition. 


\section{Materials and Methods}

The field experiment was carried out to study the pre and post-emergence herbicides with cultural practices on weed management in soybean - pigeonpea intercropping system under middle Gujarat condition. The experiment was conducted at Agriculture Research Station, Anand Agricultural University, Derol, Dist: Panchmahal, Gujarat, India during kharif seasons of the years 2017-18 and 201819.The ten weed control treatments were studied viz.,pendimethalin $1000 \mathrm{~g} \mathrm{ha}^{-1} \mathrm{PE} f b$ interculturing + hand weeding at $30 \mathrm{DAS}$, clomazone $1000 \mathrm{~g} \mathrm{ha}^{-1} \mathrm{PE} f b$ interculturing + hand weeding at 30 DAS, imazethapyr $75 \mathrm{~g}$ $\mathrm{ha}^{-1} \mathrm{PoE} f b$ interculturing + hand weeding at 30 DAS, propaquizafop $75 \mathrm{~g} \mathrm{ha}^{-1} \mathrm{PoE} f b$ interculturing + hand weeding at $30 \mathrm{DAS}$, quizalofop ethyl $50 \mathrm{~g} \mathrm{ha}^{-1} \mathrm{PoE} f b$ interculturing + hand weeding at 30 DAS,fenoxaprop-p-ethyl $100 \mathrm{~g} \mathrm{ha}^{-1} \mathrm{PoE}$ $f b$ interculturing + hand weeding at $30 \mathrm{DAS}$, imazamox $35 \%$ + imazethapyr $35 \%$ (Pre mix ) $70 \mathrm{~g} \mathrm{ha}^{-1} \mathrm{PoE} f b$ interculturing + hand weeding at 30 DAS, sodium acefluorfen $16.5 \%+$ clodinafop propargyl 8\% EC (Pre-mix) $80+165$ PoE $f b$ interculturing + hand weeding at $30 \mathrm{DAS}$, interculturing + hand weeding at $20 \& 40$ DAS and weedy check. The experiment was laid out in Randomized Block design with four replications. The plot size was $3.6 \times 6.0 \mathrm{~m}$. The soil of the experimental field was sandy loam in texture having low in organic carbon and nitrogen, medium in available phosphorus and high in potassium. Pendimethalin and clomazone was applied as pre emergence while imazethapyr, propaquizafop, quizalofop ethyl, fenoxaprop-p-ethyl, imazamox $35 \%$ + imazethapyr 35\% and sodium acefluorfen $16.5 \%$ + clodinafop propargyl $8 \%$ EC were applied as post emergence with flat fan nozzle using 500 litre water per hectare. The soybean variety NRC 37 and pigeonpea variety AGT 2 were used in the experiment as a test crop. All the recommended agronomic practices were followed for raising the crop during both the years of experimentation. The observations on weed count, weed dry weight and weed control efficiency was recorded. The seed and stover equivalent yield was recorded from the prevailing market price on the basis of pooled yield data and benefit cost ratio were calculated. Weed data were square-root transformed before statistical analysis. Pooled analysis of two years was done and comparison were made at $5 \%$ level of significance.

\section{Results and Discussion \\ Weed flora}

The experimental field was infested with Echinochloa crusgalli L.,Eragrostis major L. Digitaria sanguinalis L.and Cynodon Dactylon L. as a monocot weeds (48.6\%), while Digera arvensis L.,Phyllanthus niruri L. and Launaea mudicauli L. as a dicot weeds (37.2\%) and Cyperus Rotundus L. as a sedge weed (14.1\%), were the major weeds associated with the soybean and pigeonpea crop. Govind Prasad (2008) ${ }^{[4]}$ also reported similar weed flora in soybean and Sing and Shekhon (2013) ${ }^{[16]}$ in pigeonpea.

\section{Effect on weed density and weed dry weight}

The pooled data of two years presented in (Table 1) indicated that, all the weed control treatments significantly reduced the weeds count and weed dry weight when compared with unweeded control. Monocot weeds count was recorded at 40 DAS. Significantly less number of monocot weeds (1.00 no. $\left.\mathrm{m}^{-2}\right)$ was recorded by the treatment $\mathrm{T}_{9}(\mathrm{IC}+\mathrm{HW}$ at $20 \&$ 40 DAS). The next best treatment was $\mathrm{T}_{7}$ (imazamox $35 \%+$ imazethapyr $35 \%$ (Pre mix) $70 \mathrm{~g} \mathrm{ha}^{-1} \mathrm{PoE} f b \mathrm{IC}+\mathrm{HW}$ at 30
DAS) (3.42 no.m ${ }^{-2}$ ), which was statistically at par with all other treatments except treatment $\mathrm{T}_{10}$ (weedy check). Significantly the maximum number of monocot weeds $(6.76$ no. $\mathrm{m}^{-2}$ ) was recorded under treatment $\mathrm{T}_{10}$ (weedy check). Monocot weeds count (no. $\mathrm{m}^{-2}$ ) was recorded at harvest of soybean. Significantly less number of monocot weeds (3.35 no. $\left.\mathrm{m}^{-2}\right)$ was recorded by the treatment $\mathrm{T}_{9}(\mathrm{IC}+\mathrm{HW}$ at $20 \&$ 40 DAS). The next best treatment was $\mathrm{T}_{3}$ (imazethapyr $75 \mathrm{~g}$ $\mathrm{ha}^{-1} \mathrm{PoE} f b \mathrm{IC}+\mathrm{HW}$ at $\left.30 \mathrm{DAS}\right)\left(4.37\right.$ no.m $\left.^{-2}\right)$, which was statistically at par with all other treatments except treatment $\mathrm{T}_{10}$ (weedy check). Significantly the maximum number of monocot weeds $\left(9.16\right.$ no. $^{-2}$ ) was observed under treatment $\mathrm{T}_{10}$ (weedy check). Similar, findings were also reported by Pandya et al. (2007) ${ }^{[14]}$ and Govind Prasad (2008) ${ }^{[4]}$ in soybean and Jadhav (2015) ${ }^{[7]}$ in soybean + pigeonpea intercropping.

Dicot weeds count $\left(\right.$ no. $\mathrm{m}^{-2}$ ) was recorded at 40 DAS. Significantly less number of dicot weeds $\left(1.00\right.$ no. $\left.\mathrm{m}^{-2}\right)$ was recorded by the treatment $\mathrm{T}_{9}$ (IC $+\mathrm{HW}$ at $\left.20 \& 40 \mathrm{DAS}\right)$. The next best treatment was $\mathrm{T}_{7}$ (imazamox $35 \%+$ imazethapyr $35 \%$ (Pre mix) $70 \mathrm{~g} \mathrm{ha}^{-1} \mathrm{PoE} f b \mathrm{IC}+\mathrm{HW}$ at $\left.30 \mathrm{DAS}\right)(3.43$ no. $\left.\mathrm{m}^{-2}\right)$, which was statistically at par with all other treatments except treatment $\mathrm{T}_{1}$ (pendimethalin $1000 \mathrm{~g} \mathrm{ha}^{-1} \mathrm{PE} f b \mathrm{IC}+\mathrm{HW}$ at $30 \mathrm{DAS}$ ), $\mathrm{T}_{2}$ (clomazone $1000 \mathrm{~g} \mathrm{ha}^{-1} \mathrm{PE} f b \mathrm{IC}+\mathrm{HW}$ at 30 DAS) andT 10 (weedy check). Significantly the maximum number of dicot weeds $\left(5.89\right.$ no. $\left.^{-2}\right)$ was observed under treatment $\mathrm{T}_{10}$ (weedy check). Dicot weeds count (no. $\mathrm{m}^{-2}$ ) was recorded at harvest of soybean. Significantly less number of dicot weeds $\left(3.84\right.$ no. $\left.\mathrm{m}^{-2}\right)$ was recorded by the treatment $\mathrm{T}_{9}$ $(\mathrm{IC}+\mathrm{HW}$ at $20 \& 40 \mathrm{DAS})$. The next best treatment was $\mathrm{T}_{7}$ (imazamox 35\% + imazethapyr 35\% (Pre mix ) $70 \mathrm{~g} \mathrm{ha}^{-1} \mathrm{PoE}$ $f b \mathrm{IC}+\mathrm{HW}$ at $30 \mathrm{DAS})\left(4.46\right.$ no. $\left.^{-2}\right)$, which was statistically at par with treatments $\mathrm{T}_{3}$ (imazethapyr $75 \mathrm{~g} \mathrm{ha}^{-1} \mathrm{PoE} f b \mathrm{IC}+$ $\mathrm{HW}$ at $30 \mathrm{DAS}$ ) (4.74 no.m ${ }^{-2}$ ) and $\mathrm{T}_{4}$ (propaquizafop $75 \mathrm{~g} \mathrm{ha}^{-}$ ${ }^{1} \mathrm{PoE} f b \mathrm{IC}+\mathrm{HW}$ at 30 DAS) (4.94 no.m $\left.{ }^{-2}\right)$.Significantly the maximum number of dicot weeds $\left(8.56\right.$ no. $\left.\mathrm{m}^{-2}\right)$ was observed under treatment $\mathrm{T}_{10}$ (weedy check). Similar findings were obtained by application of imazethapyr 35\%+imazamox 35\% $75,87.5 \mathrm{~g} \mathrm{ha}^{-1}$ and imazamox $\left(350 \mathrm{ml} \mathrm{ha}^{-1}\right)$ as early postemergence produced better yield attributing characters compared to weedy check an account of maximum reduction in weed growth coupled with no inhibitory effects on soybean plants reported by Kothawade et al. (2007) ${ }^{[8]}$ and Govind prasad (2008) [4] whereas similar findings reported in soybean+ pigeonpea intercropping by Jadhav (2015) ${ }^{[7]}$.

Sedge weeds count $\left(\right.$ no.m $\left.^{-2}\right)$ was recorded at 40 DAS. Significantly less number of sedge weeds $\left(1.00\right.$ no.m $\left.^{-2}\right)$ was recorded by the treatment $\mathrm{T}_{9}(\mathrm{IC}+\mathrm{HW}$ at $20 \& 40 \mathrm{DAS})$. The next best treatment was $\mathrm{T}_{7}$ (imazamox $35 \%+$ imazethapyr $35 \%$ (Pre mix ) $70 \mathrm{~g} \mathrm{ha}^{-1} \mathrm{PoE} f b \mathrm{IC}+\mathrm{HW}$ at $\left.30 \mathrm{DAS}\right)(2.73$ no. $\mathrm{m}^{-2}$ ), which was not statistically at par with all other treatments. Significantly the maximum number of sedge weeds $\left(3.73\right.$ no. $\left.^{-2}\right)$ was recorded under treatment $T_{10}$ (weedy check). Sedge weeds count (no. $\mathrm{m}^{-2}$ ) was recorded at harvest of soybean. Significantly less number of sedge weeds $(2.85$ no. $\left.\mathrm{m}^{-2}\right)$ was recorded by the treatment $\mathrm{T}_{9}(\mathrm{IC}+\mathrm{HW}$ at $20 \&$ 40 DAS). The next best treatments $\mathrm{T}_{7}$ (imazamox $35 \%+$ imazethapyr $35 \%$ (Pre mix ) $70 \mathrm{~g} \mathrm{ha}^{-1} \mathrm{PoE} f b \mathrm{IC}+\mathrm{HW}$ at 30 DAS) $\left(3.14\right.$ no. $\left.^{-2}\right)$, which was statistically at par with $\mathrm{T}_{3}$ (imazethapyr $75 \mathrm{~g} \mathrm{ha}^{-1} \mathrm{PoE} f b \mathrm{IC}+\mathrm{HW}$ at $\left.30 \mathrm{DAS}\right)(3.29$ no. $\mathrm{m}^{-2}$ ) and $\mathrm{T}_{4}$ (propaquizafop $75 \mathrm{~g} \mathrm{ha}^{-1} \mathrm{PoE} f b \mathrm{IC}+\mathrm{HW}$ at 30 DAS) (3.32 no. $\left.\mathrm{m}^{-2}\right)$, except all other treatments. Significantly the maximum number of sedge weeds was $(5.13$ no. $\mathrm{m}^{-2}$ ) observed under treatment $\mathrm{T}_{10}$ (weedy check). Similar 
result was opined by Pandya et al. (2007) ${ }^{[14]}$ and Habimana et al. ${ }^{[5]}$ (2013).

Total weeds count $\left(\right.$ no. $\left.\mathrm{m}^{-2}\right)$ was recorded at 40 DAS. Significantly less number of total weeds was $\left(1.00\right.$ no. $\left.^{-2}\right)$ recorded by the treatment $\mathrm{T}_{9}(\mathrm{IC}+\mathrm{HW}$ at $20 \& 40 \mathrm{DAS})$. The next best treatment was $\mathrm{T}_{7}$ (imazamox $35 \%+$ imazethapyr $35 \%$ (Pre mix ) $70 \mathrm{~g} \mathrm{ha}^{-1} \mathrm{PoE} f b \mathrm{IC}+\mathrm{HW}$ at $\left.30 \mathrm{DAS}\right)(5.39$ no. $\mathrm{m}^{-2}$ ), which was statistically at par with $\mathrm{T}_{3}$ (imazethapyr 75 $\mathrm{g} \mathrm{ha}^{-1}$ PoE $f b$ IC $+\mathrm{HW}$ at $\left.30 \mathrm{DAS}\right)\left(5.64\right.$ no. $\left.\mathrm{m}^{-2}\right)$ except all other treatments Significantly the maximum number of total weeds $\left(9.63\right.$ no. $^{-2}$ ) was recorded under treatment $T_{10}$ (weedy check). Total weeds count $\left(\right.$ no. $\left.\mathrm{m}^{-2}\right)$ was recorded at harvest of soybean. Significantly less number of total weeds $\left(5.67\right.$ no.m ${ }^{-}$ ${ }^{2}$ ) was recorded by the treatment $\mathrm{T}_{9}(\mathrm{IC}+\mathrm{HW}$ at $20 \& 40$ DAS). The next best treatment was $\mathrm{T}_{7}$ (imazamox $35 \%+$ imazethapyr 35\% (Pre mix ) $70 \mathrm{~g} \mathrm{ha}^{-1} \mathrm{PoE} f b \mathrm{IC}+\mathrm{HW}$ at 30 DAS) $\left(6.86\right.$ no. $\left.^{-2}\right)$, which was statistically at par withT $T_{3}$ (imazethapyr $75 \mathrm{~g} \mathrm{ha}^{-1} \mathrm{PoE} f b \mathrm{IC}+\mathrm{HW}$ at $\left.30 \mathrm{DAS}\right)(7.12$ no. $\mathrm{m}^{-2}$ ) and $\mathrm{T}_{4}$ (propaquizafop $75 \mathrm{~g} \mathrm{ha}^{-1} \mathrm{PoE} f b \mathrm{IC}+\mathrm{HW}$ at 30 DAS)(7.31 no. $\left.\mathrm{m}^{-2}\right)$, except all other treatments. Significantly the maximum number of total weeds $\left(13.57\right.$ no.m $\left.^{-2}\right)$ was observed under treatment $\mathrm{T}_{10}$ (weedy check). Similar result was opined by Pandya et al. (2007) ${ }^{[14]}$ and Kundu et al. (2011) ${ }^{[9]}$ in soybean.

The minimum numbers of weeds was obtained under treatment $\mathrm{T}_{9}(\mathrm{IC}+\mathrm{HW}$ at $20 \& 40 \mathrm{DAS})$ at $40 \mathrm{DAS}$ and at harvest this was evident due to practically least weed emergence after cultural operation. The minimum weed population recorded under these treatments was due to efficiency of herbicides to control weeds at early stage and later on through weeding and interculturing operation done at 40 DAS cause the least emergence of weeds and hence, minimum weed population. In addition to dense crop might have smothering effect on weeds. These finding corroborate the result reported by Pandya et al. (2007) ${ }^{[14]}$, Kundu et al. (2011) ${ }^{[9]}$ and Habimana et al. (2013) ${ }^{[5]}$ in soybean, whereas similar findings reported in soybean+ pigeonpea intercropping by Jadhav (2015) ${ }^{[7]}$.

Results on dry weight of weeds recorded at harvest soybean were significantly influenced due to different weed management practices. Significantly less number of monocot weeds dry weight $\left(2.35 \mathrm{~g} \mathrm{~m}^{-2}\right)$ was recorded by the treatment $\mathrm{T}_{9}$ (IC + HW at $\left.20 \& 40 \mathrm{DAS}\right)$. The next best treatment was $\mathrm{T}_{7}$ (imazamox $35 \%$ + imazethapyr $35 \%$ (Pre mix) $70 \mathrm{~g} \mathrm{ha}^{-}$ ${ }^{1} \mathrm{PoE} f b \mathrm{IC}+\mathrm{HW}$ at $\left.30 \mathrm{DAS}\right)\left(3.05 \mathrm{~g} \mathrm{~m}^{-2}\right)$, which was statistically at par with all other treatments except treatment $\mathrm{T}_{10}$ (weedy check). Significantly the maximum number of monocot weeds dry weight $\left(6.42 \mathrm{~g} \mathrm{~m}^{-2}\right)$ was observed under treatment $\mathrm{T}_{10}$ (weedy check). Significantly less number of dicot weeds dry weight $\left(2.74 \mathrm{~g} \mathrm{~m}^{-2}\right)$ was recorded by the treatment $\mathrm{T}_{9}(\mathrm{IC}+\mathrm{HW}$ at $20 \& 40$ DAS)which was statistically at par with treatment $\mathrm{T}_{7}$ (imazamox $35 \%+$ imazethapyr $35 \%$ (Pre mix ) $70 \mathrm{~g} \mathrm{ha}^{-1} \mathrm{PoE} f b \mathrm{IC}+\mathrm{HW}$ at 30 DAS) (2.94 $\left.\mathrm{gm}^{-2}\right)$, except all other treatments Significantly the maximum number of dicot weeds dry weight $\left(10.10 \mathrm{~g} \mathrm{~m}^{-2}\right)$ was observed under treatment $\mathrm{T}_{10}$ (weedy check).Significantly less number of sedge weeds dry weight $\left(1.62 \mathrm{~g} \mathrm{~m}^{-2}\right)$ was recorded by the treatment $\mathrm{T}_{9}(\mathrm{IC}+\mathrm{HW}$ at 20 \& 40 DAS) which was statistically at par with treatment $\mathrm{T}_{7}$ (imazamox 35\% + imazethapyr 35\% (Pre mix) $70 \mathrm{~g} \mathrm{ha}^{-1} \mathrm{PoE}$ $f b \mathrm{IC}+\mathrm{HW}$ at $30 \mathrm{DAS})\left(1.65 \mathrm{~g} \mathrm{~m}^{-2}\right), \mathrm{T}_{3}$ (imazethapyr $75 \mathrm{~g} \mathrm{ha}^{-}$ $1 \mathrm{PoE} f b \mathrm{IC}+\mathrm{HW}$ at $30 \mathrm{DAS})\left(1.69 \mathrm{~g} \mathrm{~m}^{-2}\right)$ and $\mathrm{T}_{4}$ (propaquizafop $75 \mathrm{~g} \mathrm{ha}^{-1} \mathrm{PoE} f b \mathrm{IC}+\mathrm{HW}$ at $\left.30 \mathrm{DAS}\right)(1.73 \mathrm{~g}$ $\mathrm{m}^{-2}$ ). Significantly the maximum number of sedge weeds dry weight was observed under treatment $\mathrm{T}_{10}$ (weedy check).
Significantly less number of total weeds dry weight $\left(3.71 \mathrm{~g} \mathrm{~m}^{-}\right.$ $\left.{ }^{2}\right)$ was recorded by the treatment $\mathrm{T}_{9}(\mathrm{IC}+\mathrm{HW}$ at $20 \& 40$ DAS) The next best treatment was $\mathrm{T}_{7}$ (imazamox $35 \%+$ imazethapyr $35 \%$ (Pre mix) $70 \mathrm{~g} \mathrm{ha}^{-1} \mathrm{PoE} f b \mathrm{IC}+\mathrm{HW}$ at 30 DAS) (4.32 $\left.\mathrm{g} \mathrm{m}^{-2}\right)$,which was statistically at par with treatment $\mathrm{T}_{3}$ (imazethapyr $75 \mathrm{~g} \mathrm{ha}^{-1} \mathrm{PoE} f b \mathrm{IC}+\mathrm{HW}$ at 30 DAS) $\left(4.53 \mathrm{~g} \mathrm{~m}^{-2}\right)$. Significantly the maximum number of total weeds dry weight $\left(12.36 \mathrm{~g} \mathrm{~m}^{-2}\right)$ was observed under treatment $\mathrm{T}_{10}$ (weedy check). Similar result was opined by Pandya et al. (2007) [14], Kundu et al. (2011) [9], Habimana et al. (2013) [5] and Upadhyay et al. (2013) [20] in soybean, whereas similar findings reported in soybean + pigeonpea intercropping by Jadhav (2015) [7]. The results of the experiments showed that, dry matter of weeds in weedy check was maximum because of higher weed intensity and higher dry weight due to its dominance in utilizing the growth resources like sunlight, nutrients, moisture, $\mathrm{CO}_{2}$ etc., weed free check recorded significantly lower dry weight at all the stages of pigeonpea. These result are in close conformity with those reported by Dhonde et al. (2009) ${ }^{[3]}$, Sukhadia et al. (2000) ${ }^{[18]}$ and Rao et al. $(2015)^{[21]}$.

\section{Weed control efficiency $(\%)$}

The Data (Table 1) showed that weed control efficiency $(91.9 \%)$ was recorded maximumin the treatment $\mathrm{T}_{9}$ (IC $+\mathrm{HW}$ at $20 \& 40 \mathrm{DAS}(91.9 \%)$ followed by $\mathrm{T}_{7}$ (imazamox $35 \%+$ imazethapyr $35 \%$ (Pre mix) $70 \mathrm{~g} \mathrm{ha}^{-1} \mathrm{PoE} f b \mathrm{IC}+\mathrm{HW}$ at 30 DAS) $\left(88.8 \%\right.$ ) and treatment $\mathrm{T}_{3}$ (imazethapyr $75 \mathrm{~g} \mathrm{ha}^{-1} \mathrm{PoE} f b$ $\mathrm{IC}+\mathrm{HW}$ at $30 \mathrm{DAS})(87.7 \%)$. Weed control efficiency was recorded higher in interculturing + two hand weeding at $20 \&$ 40 DAS followed by application of imazamox $35 \%+$ imazethapyr $35 \%$ (Pre mix ) $70 \mathrm{~g} \mathrm{ha}^{-1} \mathrm{PoE} f b \mathrm{IC}+\mathrm{HW}$ at 30 DAS and application of imazethapyr $75 \mathrm{~g} \mathrm{ha}^{-1} \mathrm{PoE} f b$ IC + $\mathrm{HW}$ at $30 \mathrm{DAS}$, were shown very effective in controlling broad leaf, grasses and sedges have arrested growth of most of the weeds, resulting in higher weed control efficiency and thus allowed the crop to grow more vigorously. Similar result was opined by Pandya et al. (2007) [14], Upadhyay et al. (2012) ${ }^{[20]}$ and Habimana et al. (2013) ${ }^{[5]}$ in soybean crop, whereas similar findings reported in soybean + pigeonpea intercropping by Jadhav (2015) ${ }^{[7]}$.

\section{Effect on seed and stover yield}

Data regarding equivalent seed and stover yield $\left(\mathrm{kg} \mathrm{ha}^{-1}\right)$ of soybean was recorded as influenced by weed management practices are presented in (Tables 2). The higher equivalent seed and stover yield of $\left(3641 \mathrm{~kg} \mathrm{ha}^{-1}\right.$ and $\left.4944 \mathrm{~kg} \mathrm{ha}^{-1}\right)$ was recorded under treatment $\mathrm{T}_{9}$ (IC $+\mathrm{HW}$ at $\left.20 \& 40 \mathrm{DAS}\right)$, which was statistically at par with treatment $\mathrm{T}_{7}$ (imazamox $35 \%$ + imazethapyr $35 \%$ (Pre mix) $70 \mathrm{~g} \mathrm{ha}^{-1} \mathrm{PoE} f b \mathrm{IC}+\mathrm{HW}$ at 30 DAS) (3380 kg ha-1 and $\left.4584 \mathrm{~kg} \mathrm{ha}^{-1}\right)$. Significantly lowest equivalent seed and stover yield of $\left(986 \mathrm{~kg} \mathrm{ha}^{-1}\right.$ and $1277 \mathrm{~kg} \mathrm{ha}^{-1}$ ) was recorded by the treatment $\mathrm{T}_{10}$ (weedy check). The soybean-equivalent yield was worked out considering price of soybean and pigeonpea. The observation on SEY kg ha-1 indicated that significant difference due to various treatments. Interculturing + hand weeding at $20 \& 40$ DAS recorded significantly higher SEY than all other treatments except application of imazamox $35 \%+$ imazethapyr $35 \%$ (Pre mix ) $70 \mathrm{~g} \mathrm{ha}^{-1} \mathrm{PoE} f b \mathrm{IC}+\mathrm{HW}$ at 30 DAS. The lowest SEY recorded in weedy check. Similar result was reported by Kushwah and Vyas (2005) ${ }^{[11]}$ in soybean. Jadhav (2015) [7] and Ilhe et al. (2017) [6] in soybean-pigeonpea. 


\section{Economics}

Data on economics of the different treatments presented in (Table 2) indicated maximum gross return and net return ( 114186 and $59999 \mathrm{ha}^{-1}$ ) was achieved under treatment $\mathrm{T}_{9}$ (IC $+\mathrm{HW}$ at $20 \& 40 \mathrm{DAS}$ ) and $\mathrm{T}_{7}$ (imazamox $35 \%+$ imazethapyr $35 \%$ (Pre mix) $70 \mathrm{~g} \mathrm{ha}^{-1} \mathrm{PoE} f b \mathrm{IC}+\mathrm{HW}$ at 30 DAS) ( 105971 and $56351 \mathrm{ha}^{-1}$ ). The benefit cost ratio was also higher in $\mathrm{T}_{7}$ (imazamox $35 \%+$ imazethapyr $35 \%$ (Pre mix ) $70 \mathrm{~g} \mathrm{ha}^{-1} \mathrm{PoE} f b \mathrm{IC}+\mathrm{HW}$ at $\left.30 \mathrm{DAS}\right)$ (2.14) followed by treatment $\mathrm{T}_{9}(\mathrm{IC}+\mathrm{HW}$ at $20 \& 40 \mathrm{DAS})$ (2.11). The treatment $\mathrm{T}_{10}$ (weedy check) had lowest benefit cost ratio $(0.75)$ due to poor yield in this treatment. The results are in conformity with the results of Mishra et al. (2013) [12], Upadhyay et al. (2012) ${ }^{[20]}$ and Moghal et al. (2014) ${ }^{[13]}$.

Table 1: Weed density (no. $\left.\mathrm{m}^{-2}\right)$ at 40 DAS and harvest, weed dry weight $\left(\mathrm{g} \mathrm{m}^{-2}\right)$ at harvest and weed control efficiency $(\%)$ as influenced by different weed control treatments (pooled of two year)

\begin{tabular}{|c|c|c|c|c|c|c|c|c|c|c|c|c|c|c|}
\hline \multirow{2}{*}{$\begin{array}{l}\text { Tr. } \\
\text { No. }\end{array}$} & \multirow[t]{2}{*}{ Treatment } & \multicolumn{4}{|c|}{ Weed density $\left(\right.$ no. $\left.\mathrm{m}^{-2}\right)$ at 40 DAS } & \multicolumn{4}{|c|}{ Weed density $\left(\right.$ no. $\left.\mathrm{m}^{-2}\right)$ at harvest } & \multicolumn{4}{|c|}{$\begin{array}{c}\text { Weed dry weight }\left(\mathrm{g} \mathrm{m}^{-2}\right) \text { at } \\
\text { harvest }\end{array}$} & \multirow{2}{*}{$\begin{array}{l}\text { WCE } \\
(\%)\end{array}$} \\
\hline & & Monocot & Dicot & Sedges & Total & Monocot & Dicot & Sedges & Total & Monocot & Dicot & Sedges & \begin{tabular}{|l|} 
Total \\
\end{tabular} & \\
\hline $\mathrm{T}_{1}$ & $\begin{array}{c}\text { Pendimethalin } 1000 \mathrm{~g} \mathrm{~h}^{-1} \mathrm{PE} f b \mathrm{IC}+ \\
\mathrm{HW} \text { at } 30 \text { DAS }\end{array}$ & & & & & & & & & $\begin{array}{c}3.31^{\mathrm{b}} \\
(10.06) \\
\end{array}$ & $\begin{array}{c}3.57^{\mathrm{b}} \\
(11.81) \\
\end{array}$ & & & 85.1 \\
\hline $\mathrm{T}_{2}$ & $\begin{array}{c}\text { Clomazone } 1000 \mathrm{~g} \mathrm{ha}^{-1} \mathrm{PE} f b \mathrm{IC}+\mathrm{HW} \\
\text { at } 30 \mathrm{DAS}\end{array}$ & $\begin{array}{c}3.51^{\mathrm{b}} \\
(11.38)\end{array}$ & $\begin{array}{c}3.98^{\mathrm{bc}} \\
(15.00) \\
\end{array}$ & $\begin{array}{l}3.09^{\mathrm{bc}} \\
(8.63) \\
\end{array}$ & $\begin{array}{l}5.99^{\mathrm{b}} \\
(35.0) \\
\end{array}$ & $\begin{array}{c}4.46^{\mathrm{b}} \\
(19.25)\end{array}$ & & $\begin{array}{c}3.58^{\mathrm{b}} \\
(11.88)\end{array}$ & $\begin{array}{l}7.76^{\mathrm{b}} \\
(59.5) \\
\end{array}$ & $\begin{array}{l}3.27^{\mathrm{b}} \\
(9.85)\end{array}$ & $\begin{array}{c}3.50^{\mathrm{b}} \\
(11.40)\end{array}$ & & & 85.2 \\
\hline $\mathrm{T}_{3}$ & $\begin{array}{r}\text { Imazethapyr } 75 \mathrm{~g} \mathrm{ha}^{-1} \\
\text { at } 30 \mathrm{D}\end{array}$ & & & $\begin{array}{l}2.93^{\text {cd }} \\
(7.63) \\
\end{array}$ & \begin{tabular}{|l|}
$5.64^{\mathrm{bc}}$ \\
$(30.9)$ \\
\end{tabular} & $\begin{array}{c}4.37^{\mathrm{b}} \\
(18.38) \\
\end{array}$ & & & & $\begin{array}{l}3.12^{\mathrm{b}} \\
(8.87)\end{array}$ & $\begin{array}{l}3.14^{\mathrm{b}} \\
(8.90) \\
\end{array}$ & & $\begin{array}{l}4.53^{\mathrm{b}} \\
(19.6) \\
\end{array}$ & 87.7 \\
\hline $\mathrm{T}_{4}$ & $\begin{array}{r}\text { Propaquizafop } \\
\text { HW }\end{array}$ & & & $\begin{array}{l}3.01^{\mathrm{bc}} \\
(8.13)\end{array}$ & $\begin{array}{l}5.74^{\mathrm{bc}} \\
(32.0) \\
\end{array}$ & $\begin{array}{c}4.44^{\mathrm{b}} \\
(18.88)\end{array}$ & & & $\begin{array}{l}7.31^{\mathrm{b}} \\
(52.6)\end{array}$ & $\begin{array}{l}3.1 \\
(9.1\end{array}$ & $\begin{array}{c}3.37^{\mathrm{b}} \\
(10.51)\end{array}$ & & & 86.5 \\
\hline $\mathrm{T}_{5}$ & $\begin{array}{r}\text { Quizalofop et } \\
\mathrm{H} \\
\end{array}$ & & & $\begin{array}{l}3.16^{\mathrm{b}} \\
(9.00) \\
\end{array}$ & \begin{tabular}{|l|}
$5.87^{\mathrm{bc}}$ \\
$(33.5)$ \\
\end{tabular} & $\begin{array}{c}4.56^{\mathrm{b}} \\
(19.93) \\
\end{array}$ & & & & & & & & 85.6 \\
\hline $\mathrm{T}_{6}$ & $\begin{array}{r}\text { Fenoxaprop - } \\
\text { IC + }\end{array}$ & $\begin{array}{l}3.69^{\mathrm{b}} \\
(12.75)\end{array}$ & & $\begin{array}{l}3.15^{\mathrm{b}} \\
(9.00) \\
\end{array}$ & \begin{tabular}{|l|}
$5.98^{\mathrm{b}}$ \\
$(34.8)$ \\
\end{tabular} & $\begin{array}{l}4.65^{\mathrm{b}} \\
(20.75)\end{array}$ & & $\begin{aligned} 3.5 \\
(11 .\end{aligned}$ & $\begin{array}{l}7.71^{\mathrm{b}} \\
(58.5)\end{array}$ & $\begin{array}{l}3.22^{\mathrm{b}} \\
(9.41)\end{array}$ & $\begin{array}{c}3.44^{\mathrm{b}} \\
(11.04) \\
\end{array}$ & & $1^{\mathrm{b}}$ & 85.5 \\
\hline $\mathrm{T}_{7}$ & $\begin{array}{r}\text { Imazamox } 35 \%+ \\
(\text { Pre mix ) } \\
f b \text { IC }+ \text { HW } \\
\end{array}$ & & & & & & & & & & & & & 88.8 \\
\hline $\mathrm{T}_{8}$ & $\begin{array}{c}\text { Sodium acefluorfen } 16.5 \%+ \\
\text { Clodinafop propargyl } 8 \% \text { EC (Pre-mix) } \\
80+165 \text { PoE } \\
f b \text { IC }+ \text { HW at 30 DAS }\end{array}$ & $\begin{array}{c}3.58^{\mathrm{b}} \\
(11.88)\end{array}$ & $\begin{array}{c}3.67^{\mathrm{bc}} \\
(12.50)\end{array}$ & $\begin{array}{l}3.14^{\mathrm{bc}} \\
(8.88)\end{array}$ & $\mid \begin{array}{l}5.85^{\mathrm{bc}} \\
(33.3)\end{array}$ & $\begin{array}{c}4.57^{\mathrm{b}} \\
(20.00)\end{array}$ & $\begin{array}{l}5.11^{\mathrm{b}} \\
(25.3)\end{array}$ & $\begin{array}{c}3.44^{\mathrm{bc}} \\
(10.88)\end{array}$ & $\begin{array}{c}7.55^{\mathrm{b}} \\
(56.1)\end{array}$ & $\begin{array}{c}3.19^{\mathrm{b}} \\
(9.24)\end{array}$ & $\begin{array}{c}3.34^{\mathrm{b}} \\
(10.20)\end{array}$ & $\begin{array}{l}1.74^{\text {cde }} \\
(2.05)\end{array}$ & $\begin{array}{c}4.73^{\mathrm{b}} \\
(21.5)\end{array}$ & 86.6 \\
\hline $\mathrm{T}_{9}$ & IC + HW at $20 \& 40$ DAS & & & & $\begin{array}{l}1.00^{\mathrm{d}} \\
(0.0) \\
\end{array}$ & & & $\begin{array}{l}2.85^{\mathrm{e}} \\
(7.25)\end{array}$ & & $\begin{array}{r}2.35^{\mathrm{c}} \\
(4.61) \\
\end{array}$ & $\begin{array}{l}2.74^{\mathrm{b}} \\
(6.64) \\
\end{array}$ & $\begin{array}{l}1.62^{\mathrm{e}} \\
(1.63) \\
\end{array}$ & $\begin{array}{l}3.71^{\mathrm{b}} \\
(12.9) \\
\end{array}$ & 91.9 \\
\hline $\mathrm{T}_{10}$ & Weedy check & & $\begin{array}{c}5.89^{\mathrm{a}} \\
(34.25)\end{array}$ & $\begin{array}{c}3.73^{\mathrm{a}} \\
(13.00)\end{array}$ & $\begin{array}{c}9.63^{\mathrm{a}} \\
(92.0)\end{array}$ & $\begin{array}{c}9.16^{\mathrm{a}} \\
(84.00)\end{array}$ & $\begin{array}{r}8.56^{\mathrm{a}} \\
(79.0)\end{array}$ & $\begin{array}{c}5.13^{\mathrm{a}} \\
(25.38)\end{array}$ & $\begin{array}{c}13.57^{\mathrm{a}} \\
(188.4)\end{array}$ & $\begin{array}{c}6.42^{\mathrm{a}} \\
(40.76)\end{array}$ & $\begin{array}{c}10.10^{\mathrm{a}} \\
(110.01 \\
)\end{array}$ & $\begin{array}{l}3.18^{\mathrm{a}} \\
(9.10)\end{array}$ & $\begin{array}{c}12.36^{\mathrm{a}} \\
(159.9)\end{array}$ & - \\
\hline & & & & & 0.1 & & & & 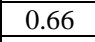 & & 0.87 & 0.04 & 0.82 & - \\
\hline & & & & & & & & & & & & & Sig. & - \\
\hline & teraction & & 0 & 0.0 & 0.12 & 0.17 & 0.18 & 0.12 & 0.17 & 0. & 0.12 & 0.06 & 0.12 & - \\
\hline & $\mathrm{FTes}$ & NS & Sig. & NS & Sig. & & & & & $\mathrm{Si}$ & Sig. & NS & Sig. & - \\
\hline & $\mathrm{CV} \%$ & 7.25 & 7.19 & 6.20 & 4.04 & 7.05 & 6.75 & 6.53 & 4.43 & 6.38 & 6.29 & 5.96 & 4.44 & - \\
\hline
\end{tabular}

Note: Data subjected to $\sqrt{ }(\mathrm{X}+1)$ transformation, figures in parenthesis are means of original values, treatment means with the letter/letters in common are not significantly different by Duncan's New Multiple Range Test at 5\% per cent level of significance

Table 2: Economics as influenced by weed management practices in soybean-pigeonpea intercropping system (pooled of two years)

\begin{tabular}{|c|c|c|c|c|c|c|c|}
\hline \multirow[b]{2}{*}{$\begin{array}{l}\text { Tr. } \\
\text { No. }\end{array}$} & \multirow[b]{2}{*}{ Treatment } & \multicolumn{2}{|c|}{$\begin{array}{c}\text { Soybean } \\
\text { equivalent yield }\end{array}$} & \multirow{2}{*}{$\begin{array}{c}\text { Gross } \\
\text { return } \\
\left(\text { ha }^{-1}\right)\end{array}$} & \multirow{2}{*}{$\begin{array}{c}\begin{array}{c}\text { Cost of } \\
\text { cultivation } \\
\left(\mathbf{h a}^{-1}\right)\end{array} \\
\text { a }\end{array}$} & \multirow{2}{*}{$\begin{array}{c}\text { Net } \\
\text { return } \\
\left(\text { (ha }^{-1}\right)\end{array}$} & \multirow{2}{*}{$\begin{array}{l}\text { B:C } \\
\text { ratio }\end{array}$} \\
\hline & & \begin{tabular}{|c|} 
Seed \\
Yield \\
$\left(\mathrm{kg} \mathrm{ha}^{-1}\right)$ \\
\end{tabular} & $\begin{array}{c}\text { Stover } \\
\text { Yield } \\
\left(\mathrm{kg} \mathrm{ha}^{-1}\right)\end{array}$ & & & & \\
\hline $\mathrm{T}_{1}$ & Pendimethalin $1000 \mathrm{~g} \mathrm{ha}^{-1} \mathrm{PE} f b \mathrm{IC}+\mathrm{HW}$ at $30 \mathrm{DAS}$ & $2708^{\mathrm{c}}$ & $3680^{\mathrm{d}}$ & 84931 & 49525 & 35406 & 1.71 \\
\hline $\mathrm{T}_{2}$ & Clomazone $1000 \mathrm{~g} \mathrm{ha}^{-1} \mathrm{PE} f b \mathrm{IC}+\mathrm{HW}$ at $30 \mathrm{DAS}$ & $2831^{\mathrm{c}}$ & $3837^{\mathrm{d}}$ & 88779 & 49232 & 39547 & 1.80 \\
\hline $\mathrm{T}_{3}$ & Imazethapyr $75 \mathrm{~g} \mathrm{ha}^{-1} \mathrm{PoE} f b \mathrm{IC}+\mathrm{HW}$ at $30 \mathrm{DAS}$ & $3261^{\mathrm{b}}$ & $4425^{\mathrm{b}}$ & 102248 & 48908 & 53340 & 2.09 \\
\hline $\mathrm{T}_{4}$ & Propaquizafop $75 \mathrm{~g} \mathrm{ha}^{-1} \mathrm{PoE} f b \mathrm{IC}+\mathrm{HW}$ at $30 \mathrm{DAS}$ & $3220^{\mathrm{b}}$ & $4370^{\mathrm{b}}$ & 100959 & 48849 & 52110 & 2.07 \\
\hline $\mathrm{T}_{5}$ & Quizalofop ethyl $50 \mathrm{~g} \mathrm{ha}^{-1} \mathrm{PoE} f b \mathrm{IC}+\mathrm{HW}$ at $30 \mathrm{DAS}$ & $3199^{\mathrm{b}}$ & $4257^{\mathrm{bc}}$ & 100238 & 49444 & 50794 & 2.03 \\
\hline $\mathrm{T}_{6}$ & Fenoxaprop -p-ethyl $100 \mathrm{~g} \mathrm{ha}^{-1} \mathrm{PoE} f b \mathrm{IC}+\mathrm{HW}$ at $30 \mathrm{DAS}$ & $2878^{\mathrm{c}}$ & $3902^{\mathrm{cd}}$ & 90245 & 48816 & 41429 & 1.85 \\
\hline $\mathrm{T}_{7}$ & Imazamox $35 \%$ + Imazethapyr 35\% (Pre mix ) $70 \mathrm{~g} \mathrm{ha}^{-1} \mathrm{PoE} f b \mathrm{IC}+\mathrm{HW}$ at $30 \mathrm{DAS}$ & $3380^{\mathrm{ab}}$ & $4584^{\mathrm{ab}}$ & 105971 & 49620 & 56351 & 2.14 \\
\hline $\mathrm{T}_{8}$ & $\begin{array}{l}\text { Sodium acefluorfen } 16.5 \%+\text { Clodinafop propargyl } 8 \% \text { EC (Pre-mix) } 80+165 \text { PoE } f b \\
\text { IC + HW at 30 DAS }\end{array}$ & $3206^{\mathrm{b}}$ & $4349^{b}$ & 100520 & 49931 & 50589 & 2.01 \\
\hline $\mathrm{T}_{9}$ & IC + HW at $20 \& 40$ DAS & $3641^{\mathrm{a}}$ & $4944^{\mathrm{a}}$ & 114186 & 54187 & 59999 & 2.11 \\
\hline $\mathrm{T}_{10}$ & Weedy check & $986^{\mathrm{d}}$ & $1277^{\mathrm{e}}$ & 30844 & 41151 & -10307 & 0.75 \\
\hline \multicolumn{8}{|c|}{$\begin{array}{ll}\text { Selling Price } \\
\end{array}$} \\
\hline \multicolumn{4}{|c|}{ Seed: Soybean:` $30.0 \mathrm{~kg}^{-1}$} & \multicolumn{4}{|c|}{ Stover: Soybean:` $1.0 \mathrm{~kg}^{-1}$} \\
\hline \multicolumn{4}{|c|}{ Pigeonpea:` $54.0 \mathrm{~kg}^{-1}$} & \multicolumn{4}{|c|}{ Pigeonpea: $` 2.0 \mathrm{~kg}^{-1}$} \\
\hline
\end{tabular}

\section{Conclusion}

From Based on the result of field experimentation, it seems quite logical that potential production, profit and economic weed management can be achieved in soybean - pigeonpea intercropping system by interculturing + hand weeding at 20 \& 40 DAS or post-emergence application of imazamox $35 \%$ + imazethapyr $35 \%$ (Pre mix) $70 \mathrm{~g} \mathrm{ha}^{-1} \mathrm{PoE} f b$ interculturing + hand weeding at 30 DAS. 


\section{References}

1. Ali M. Weed suppressing ability and productivity of short duration legumes intercropped with pigeonpea under rainfed conditions. Tropical Pest Management.1992; 34(4):384-387.

2. Bhan VM. Weeds associated with soybean and their control. Soybean production, protection and utilization. University of Illinois Urbana (Intsoy Ser.6). 1975, 147156

3. Dhonde MB, Kate SR, Pandure BS, Tambe AD. Integrated weed management in pigeonpea. Indian Journal of Weed Science. 2009; 41:102-105.

4. Govind Prasad. Integrated weed management in soybean [Glycine $\max ($ L.) Merrill] M.Sc. (agri.) Thesis (A.A.U., Anand. Gujarat), 2008.

5. Habimana S, Murthy KNK, Shankaralingappa BC, Sanjay MT, Ramachandra C. Efficiency and economics of weed control with pre and post emergence herbicides in soybean. Asian Journal of Plant Science and Research. 2013; 3(4):18-20.

6. Ilhe SS, Patil HM, Patil DB, Pawar PP, Wadile SC. Integrated weed management in soybean + pigeonpea intercropping system under rainfed. 2017; Department of Agronomy, Punjab Agricultural University, Ludhiana, Punjab, India $25^{\text {th }}$ Asian-Pacific Weed Science Society Conference on "Weed Science for Sustainable Agriculture, Environment and Biodiversity".

7. Jadhav AS. Performance of post emergence weedicide in soybean + Pigeon pea Intercropping. Journal of Agriculture and Veterinary Science. 2015; 8(7):61-62.

8. Kothawade TR, Sinare BT, Londhe TB, Shete BT. Chemical weed control in soybean. Journal of Maharashtra Agricultural University. 2007; 32(2):274275.

9. Kundu R, Brahmachari K, Bera PS, Kundu CK, Roychoudhury S. Bioefficacy of Imazethapyr on the predominant weeds in soybean. Journal of crop and weed. 2011; 7(2):173-178.

10. Kurchania SP, Rathi GS, Bhalla CS, Mathew R. Bio efficiency of Post emergence herbicides for weed control in soybean (Glycine max). Indian Journal of Weed Science. 2001; 33(1-2):34-37.

11. Kushwah SS, Vyas MD. Herbicidal weed control in soybean (Glycine max). Indian Journal of Agronomy. 2005; 50(3):225-227.

12. Mishra P, Singh Harvir, Babu Subhash, Pal Suresh. Bioefficacy of some early post emergence herbicides in soybean. Annals of Agricultural Research. 2013; 24(4):970-972.

13. Moghal HS, Kalegore NK, Kadam SR. Study of different weed control treatment on economics of soybean. In: Proceeding Biennial Conference of Indian Society of Weed Science on "Emerging Challenges in Weed Management". February 15-17, 2014. Directorate of Weed Science Research, Jabalpur, Madhya Pradesh, India, 2001, 172.

14. Pandya AK, Joshi OP, Billor SD. Effect of herbicidal weed control on weed dynamics and yield of soybean. Soybean Research. 2007; 5:26-32.

15. Sangeetha C, Chinnusamy C, Prabhakaran NK. Early post-emergence herbicides for weed control in soybean. Indian Journal of Weed Science. 2013; 45(2):140-142.

16. Singh G, Shekhon HS. Integrated weed management in pigeonpea [Cajanus cajan (L.) Millsp.]. World Journal of Agricultural Science. 2013; 9(1):86-91.
17. Sobney JS, Sindhu MS, Maran RS, Kumar K. Intercropping as a system to manage. Weed Journal of Research PAV. 1989; 26(4):563-568.

18. Sukhadia NM, Ramani BB, Modhwadia MM, Asodaria $\mathrm{KB}$. Integrated weed management in pigeonpea. Gujarat Agricultural Research Journal. 2000; 25:1-4.

19. Talnikar AS, Kadam GL, Karande DR, Jogdand PB. Integrated weed management in pigeonpea [Cajanus cajan (L.) Millsp.]. International Journal of Agricultural Sciences. 2008; 4(1):363-370.

20. Upadhyay VB, Singh A, Rawat A. Efficacy of early postemergence herbicides against associated weeds in soybean. Indian Journal of Weed Science. 2012; 44(4):73-75.

21. Venkat Rao P, Subbarami Reddy A, Koteshwara Rao Y. Effect of integrated weed management practices on growth and yield of pigeonpea. International Journal of Plant, Animal and Environmental Science. 2015; 5:124127. 